\title{
Variaciones anatómicas en el drenaje venoso del cuello
}

\author{
Anatomical variations in the venous drainage of the neck \\ Humberto Ferreira-Arquez ${ }^{1 \times \text { CvLAC }}$
}

Fecha correspondencia:

Recibido: octubre 28 de 2015.

Revisado: julio 6 de 2016.

Aceptado: septiembre 6 de 2016

Forma de citar:

Ferreira-Arquez H. Variaciones anatómicas en el drenaje venoso del cuello. Rev CES Med 2016; 30(2): 238-243.

\section{Open Access}

(c) Copyright

Licencia creative commons

Ética de publicaciones

Revisión por pares

Gestión por Open Journal System

ISSN 0120-8705

e-ISSN 2215-9177

Sobre los autores:

1. Docente Anatomía HumanaPrograma de Medicina. Coordinador Laboratorio de MorfologíaUniversidad de Pamplona. Norte de Santander- Colombia.

Comparte

7日G.

\section{Resumen}

Las venas superficiales, especialmente la vena yugular externa, son cada vez más utilizadas para canalización o procedimientos diagnósticos, terapias intravenosas, nutrición parenteral en pacientes debilitados. Las variaciones en el drenaje venoso de la cabeza y cuello son importantes no solamente para los anatomistas y radiólogos, sino también para los cirujanos. Durante una disección de rutina en el Laboratorio de Morfología de la Universidad de Pamplona en un cadáver masculino de 75 años de edad, se encontraron variaciones anatómicas e inusuales patrones de drenaje en la región de cara y cuello, las cuales fueron cuidadosamente disecadas y los detalles morfológicos fueron fotografiados; esas variaciones consistían en: ausencia unilateral de vena yugular externa. La vena lingual, la vena infrahioidea y la vena tiroidea superior tenían conexiones venosas transversas que drenaban en un arco venoso localizado entre la arteria carótida común y la arteria tiroidea superior. Este arco venoso drenaba hacia arriba en la vena facial común y hacia abajo en la vena yugular interna. El conocimiento de las variaciones anatómicas y morfológicas en las venas de cabeza y cuello es esencial para llevar a cabo procedimientos quirúrgicos exitosos en estas regiones.

Palabras clave: Variación anatómica, Vena yugular externa, Vena retromandibular, Conexiones venosas transversas, Arco venoso, Drenaje venoso cervical.

\section{Abstract}

The superficial veins, especially the external jugular vein are increasingly used for cannulation or diagnostic procedures, intravenous therapy, parenteral nutrition in debilitated patients. Variations in the venous drainage of the head and neck are important not only for anatomists and radiologists but also for surgeons. During routine dissection in the Laboratory of Morphology, University of Pamplona in a 75 year-old male cadaver in the region face and neck anatomical variations and unusual drainage patterns were found, which were dissected carefully and morphological details were photographed; these variations consisted of unilateral absence of external jugular vein. The lingual vein, the infrahyoid vein and the superior thyroid vein had transverse venous connections that drained into a venous arch located between the common carotid artery and the superior thyroid artery. This draining venous arch upward in the common facial vein and down into the internal jugular vein. Knowledge of the anatomical and morphological variations in the veins of the head and neck is essential to carry out successful surgical procedures in these regions. 
La relevancia e importancia de los variados patrones de drenaje de las venas de la cabeza y del cuello son de especial atención durante las cirugías de cabeza y cuello que involucran anastomosis microvasculares. Los varios modos de formación de la vena yugular externa han sido clásicamente descritos e ilustrados, con especial referencia a la vena facial por ser su mayor tributaria .
Keywords: Anatomical variation, External Jugular vein, Retromandibular vein, Transverse venous connections, Venous arch, Cervical venous drainage.

\section{Introducción}

La descripción anatómica estándar de las venas de la cara consiste en que la vena temporal superficial se une con la vena maxilar dentro del parénquima de la glándula parótida para formar la vena retromandibular. La vena retromandibular se divide en una rama anterior y una posterior antes de emerger a nivel del ápex de la glándula parótida. La rama anterior se une con la vena facial en sentido inferior y anterior con respecto al ángulo de la mandíbula para formar la vena facial común que a su vez drena dentro de la vena yugular interna. La rama posterior se une con la vena auricular posterior para formar la vena yugular externa, la cual pasa superficialmente con respecto al músculo esternocleidomastoideo y perfora la fascia cervical $2,5 \mathrm{cms}$ por encima del punto medio de la clavícula, drenando dentro de la vena subclavia (1).

La relevancia e importancia de los variados patrones de drenaje de las venas de la cabeza y del cuello son de especial atención durante las cirugías de cabeza y cuello que involucran anastomosis microvasculares. Los varios modos de formación de la vena yugular externa han sido clásicamente descritos e ilustrados, con especial referencia a la vena facial por ser su mayor tributaria $(2, \underline{3})$.

Las venas superficiales del cuello son usadas para canalización venosa central, monitoreo de la presión venosa central, en nutrición parenteral de pacientes debilitados, en endarterectomías carotideas; su exploración puede suministrar signos semiológicos de enfermedades cardiacas subyacentes. Durante el abordaje quirúrgico de la glándula parótida o reducción de fracturas condilares, la vena retromandibular es usada como guía para exponer las ramas del nervio facial. Las variaciones anatómicas de los vasos de cabeza y cuello llegan a ser clínicamente importantes para cirujanos orales y maxilofaciales, radiólogos, anestesiólogos (느-6).

El objetivo del presente reporte de caso es describir la ausencia unilateral de la vena yugular externa e inusual patrón de drenaje venoso a nivel cervical que fue evidenciado durante una disección de rutina en la región de cabeza y cuello, en pos de brindar un conocimiento adicional en lo concerniente a la anatomía de la región.

\section{Materiales y métodos}

Las variaciones anatómicas descritas fueron encontradas en el lado izquierdo de un cadáver masculino, de 75 años de edad, durante una disección de rutina de la región de cabeza y cuello, llevada a cabo en el laboratorio de morfología de la Universidad de Pamplona por estudiantes de pregrado de segundo semestre del Programa de medicina. La historia individual y las causas de muerte son desconocidas. Los detalles topográficos de las variaciones fueron examinados, registrados y fotografiados. La búsqueda bibliográfica de datos disponibles en la literatura científica sobre casos similares, en aras de realizar una comparación con lo encontrado en el presente reporte de caso, se realizó en las bases de datos de: Google Scholar, Pubmed, Cochrane, Ovid, Ebsco, SciVerse y SpringerLink.

\section{Resultados}

Al realizar la disección superficial anterolateral del cuello y retirar el músculo platysma se evidenció la emergencia por el borde posterior del músculo esternocleidomastoideo de las ramas sensitivas superficiales del plexo cervical (nervio occipital 
menor y nervio auricular mayor); a su vez no se observó la vena yugular externa. Por delante del músculo masetero se observó la arteria y vena facial, y por detrás la glándula salival parótida (figura 1).

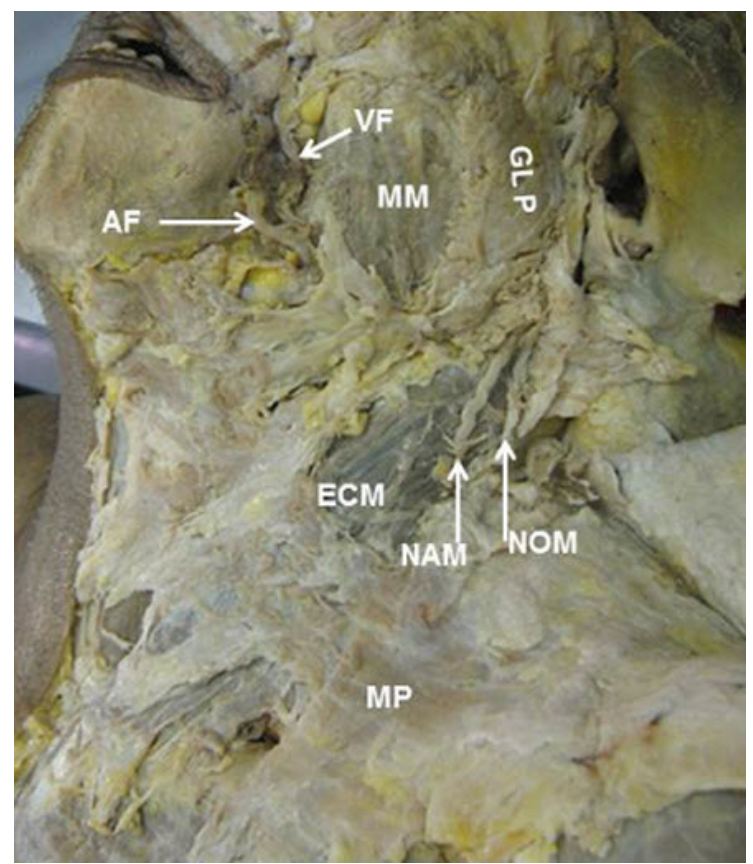

Figura 1. Vista anterolateral del cuello. Lado izquierdo- Disección superficial. MP: Músculo Platysma; ECM: Músculo sternocleidomastoideus; MM: Músculo Masseter; AF: Arteria facial; VF: Vena facial; NAM: Nervio auricular mayor, NOM: nervio occipital menor, GL P: glándula parótida

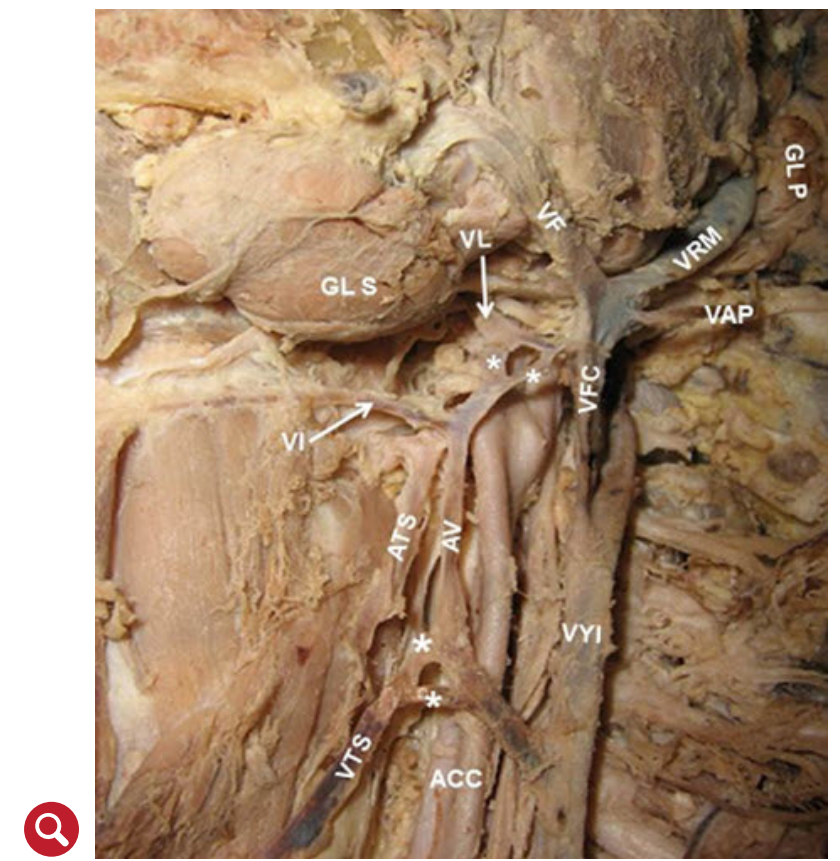

Figura 2. Vista anterolateral del cuello. Lado izquierdo. Disección profunda. VRM: Vena retromandibular; VAP: Vena auricular posterior; VF: Vena facial; VYI: Vena Yugular interna; GL S: Glándula salival submandibular; ATS: Arteria tiroidea superior; VTS: Vena tiroidea superior; VI: Vena Infrahioidea; VL: Vena lingual; VFC: Vena facial común; AV: Arco venoso; GI P: Glándula salival parótida; Asteriscos: conexiones venosas transversas.; ACC:

Arteria carótida común

Se procedió a realizar una disección más profunda para evidenciar las variaciones venosas que explicaran el drenaje venoso en ausencia de la vena yugular externa; se encontró que la vena retromandibular (VRM) no se dividía en sus ramas anterior y posterior, y presentaba un calibre inusualmente amplio.

La vena retromandibular se unía con la vena facial y formaban un tronco venoso facial común, el cual posteriormente recibía a la vena auricular posterior y terminaban drenando dentro de la vena yugular interna. También fue observado un arco venoso o canal venoso común que conectaba a la vena yugular interna y la vena facial común, en este arco venoso drenaba la vena lingual, la vena infrahioidea y la vena tiroidea superior. La vena lingual y la vena tiroidea superior se bifurcaban y drenaban a través de conexiones venosas transversas. El arco venoso fue localizado entre la arteria carótida común y la arteria tiroidea superior (figura 2).

\section{Discusión}

Las variaciones en la morfología, curso, tamaño, terminación de las venas superficiales del cuello son comunes; la formación y terminación de la vena yugular exter- 
Las variaciones en el drenaje de venas a nivel de cuello son de gran significancia en cirugías como la endarterectomía carotídea. na son variables y es difícil de determinar un patrón usual (7-9). La ausencia de vena yugular externa ha sido notificada en un $3,3 \%(\underline{10})$ y en $1 \%(\underline{11})$.

El tronco común llamado vena facial común, el cual se forma por la unión de la no dividida vena retromandibular con la vena facial, drena en la vena yugular interna a una distancia promedio de 55 - $104 \mathrm{~mm}$ desde el borde inferior del ángulo de la mandíbula, con 2 diferentes niveles que van desde 40 hasta $82 \mathrm{~mm}$ (12), en el presente estudio la distancia fue de $95 \mathrm{~mm}$.

Al respecto, ha sido notificada variación en el drenaje de la vena facial común en la vena subclavia del mismo lado (13). Algunos informes han descrito la formación de la vena yugular externa por la unión de la no dividida vena retromandibular con la vena facial, acompañando de ausencia de la vena auricular posterior, y la vena yugular externa drenando en la vena cefálica (14).

Se ha notificado la presencia de distintas formas (en $\mathrm{Y}$, en $\mathrm{U}$, en $\mathrm{U}$ alargada, en $\mathrm{N}$, en A invertida, en escalera: presentando conexiones venosas transversas) y variados tipos de canales venosos (tipo I y tipo II) o arcos venosos que conectan venas entre sí y que permiten su drenaje. Las variaciones en el drenaje de venas a nivel de cuello son de gran significancia en cirugías como la endarterectomía carotídea, donde la vaina carotidea es abierta a lo largo del borde anterior de la vena yugular interna; la vena facial común se une a la vena yugular interna justo por debajo de la bifurcación carotidea, por lo tanto suministra un adecuado y útil marcador para la localización de la bifurcación $(\underline{3}, \underline{4},-15)$.

En microcirugías reconstructivas y el diseño de colgajos cutáneos como colgajo platysma o el colgajo miocutáneo esternocleidomastoideo es de vital importancia conocer las posibles anomalías y variaciones del sistema venoso para evitar daños a la fascia que pongan en riesgo la supervivencia de los colgajos (ㅁ).

La venopunción yugular externa es un procedimiento valioso y vital, recomendado incluso más que el acceso de la vena yugular interna, de la vena subclavia o de la vena femoral; la venopunción de vasos de gran calibre representa un mayor riesgo de complicaciones intraoperativas, dentro de estas están: punción carotidea, hemorragia oculta, neumotórax y enfisema. El acceso yugular externo está indicado en aquellos pacientes donde es difícil obtener una vena periférica convencional o en pacientes donde está contraindicado canalizar un acceso venoso central, ya sea por problemas de coagulopatías o intentos fallidos en el proceso de inserción de catéteres $(\underline{3}, \underline{6})$.

El acceso yugular externo está indicado en pacientes que requieren infusión de medicamentos que no pueden pasarse por vía periférica convencional; la cateterización de la vena yugular externa se convierte en un excelente acceso a través del cual se pueden infundir hemoderivados, fármacos, electrolitos y líquidos de reposición que se deben pasar a mayor velocidad, generando menores riesgos que con los accesos venosos centrales, tales como infección y embolismo $(\underline{16}, 17)$.

Se ha descrito la cateterización de la arteria pulmonar vía vena yugular externa como un procedimiento seguro y confiable (18). Como elemento diagnóstico, una ingurgitación de la vena yugular externa refleja un aumento de la presión del circuito derecho que puede ser debida a una insuficiencia cardiaca derecha o izquierda, en su defecto a lesiones que obstruyan el flujo sanguíneo como los tumores mediastinales o pulmonares (síndrome de la vena cava superior) o incluso por trombosis de la vena cava inferior (19). 
El conocimiento de las variaciones anatómicas de la venas de cabeza y cuello es fundamental para los cirujanos y el personal de enfermería en aras de evitar errores intraoperativos, prevenir sangrados innecesarios, inapropiada disección y daños severos a estructuras anatómicas.
Existen reportes de casos individuales, unilaterales y bilaterales de variaciones anatómicas en los patrones de drenaje de las venas superficiales y profundas de la región de la cara y del cuello; sin embargo las variaciones anatómicas descritas en el presente reporte de caso son inusuales, de rara ocurrencia y descripción en la literatura científica disponible.

\section{Conclusiones}

El conocimiento de las variaciones anatómicas de la venas de cabeza y cuello es fundamental para los cirujanos y el personal de enfermería en aras de evitar errores intraoperativos, prevenir sangrados innecesarios, inapropiada disección y daños severos a estructuras anatómicas.

\section{Agradecimientos}

El autor en representación de la Universidad de Pamplona agradece al Hospital Universitario Erasmo Meoz de la Ciudad de Cúcuta por la donación de cadáveres identificados, no reclamados por familiares o personas responsables de su custodia. Proceso debidamente reglamentado, cumpliendo la normatividad legal vigente en la República de Colombia.

\section{Conflicto de interés}

Ninguno

\section{Bibliografía}

1. Selvi P, Kumar S. Variations in the venous drainage pattern of face and neck.Int J Pharm Bio Sci. 2013 Oct; 4(4): (B) 150 -154. Disponible en: http://www.ijpbs.net/ cms/php/upload/2804_pdf.pdf. Acceso en diciembre 2 de 2014

2. Shetty SD, Nayak S, Kumar N, Marpalli S, Madahv V. Unusual veins in the neck. A Case Report. Journal of Morphological Science. 2013; 30(3), 203-205. Disponible en: http://jms.org.br/PDF/v30n3a13.pdf. Acceso en diciembre 2 de 2014.

3. Gupta V, Tuli A, Choudhry R, Agarwal S, Mangal A. Facial vein draining into external jugular vein in humans: its variations, phylogenetic retention and clinical relevance. Surg Radiol Anat. 2003;25:36-41. Disponible en: https://www.ncbi.nlm. nih.gov/pubmed/12819948 Acceso en diciembre 3 de 2014.

4. Paraskevas G, Natsis K, loannidis O, Kitsoulis P, Anastasopoulos N, Spyridakis I. Multiple variations of the superficial jugular veins: case report and clinical relevance. Acta Medica (Hradec Kralove). 2014;57(1):34-7. Disponible en: http://actamedica.lfhk.cuni.cz/media/pdf/am 2014057010034.pdf. Acceso en diciembre 3 de 2014.

5. Ferreira $\mathrm{H}$. Unusual venous drainage of the head and neck. Int J Pharm Bio Sci. 2014 Oct;5 (4):(B)965-970. Disponible en: http://www.ijpbs.net/download.php?download file $=\mathrm{cms} / \mathrm{php} / \mathrm{upload} / 3797 \mathrm{pdf}$.pdf\&did=3797. Acceso en diciembre 3 de 2014.

6. Flórez JM, Arias MM, Tirado AF. Experiencia clínica de enfermería: canalización de vena yugular externa. Avances en Enfermería.2013; 31(1): 52-58. Disponible en: http://www.scielo.org.co/pdf/aven/v31n1/v31n1a06.pdf. Acceso en diciembre 2 de 2014. 
7. Bergman RA, Thompson SA, Afifi A K, Saadeh FA. Compendium of human anatomic variation: catalog, atlas and world literature. Baltimore:Urban \& Schwarzenberg. 1988

8. Choudhry R, Tuli A, Choudry S. Facial vein terminating in the external jugular vein. An embryological interpretation. Surg. Radiol. Anat. 1997;19:73-7. Disponible en: http:// link.springer.com/article/10.1007\%2FBF01628128. Acceso en diciembre 03 de 2014.

9. Kopuz C. Yavuz S. Cumhur M. Tftik S, Ilgi, S. An unusual coursing of the facial vein. Kaibogaku Zasshi. 1995;70: 20-2.

10. Brown S. The external jugular vein in American whites and negroes. Am J Phys Anthropol.1941;28(2):213-26. Disponible en http://onlinelibrary.wiley.com/ doi/10. 1002/ajpa.1330280204/pdf. Acceso en diciembre 04 de 2014.

11. Pikkieff E. On the subcutaneous veins of the neck. J Anat. 1937; 72(Pt 1): 119127. Disponible en: http://www.ncbi.nlm.nih.gov/pmc/articles/PMC1252446/ pdf/janat00526-0142.pdf. Acceso en diciembre 04 de 2014.

12. Choudhary S, Sharma AK, Singh H. Undivided retromandibular vein continuing as external jugular vein with facial vein draining into it: an anatomical variation. JK Science.2010;12(4):203-4. Disponible en: http://jkscience.org. Acceso en diciembre 04 de 2014.

13. Bertha A, Rabi S. Anatomical variations in termination of common facial vein. J Clin Diagn Res. 2011; 5: 24-27. Disponible en: http://www.jcdr.net/article fulltext.asp?id=1174. Acceso en diciembre 05 de 2014.

14. Nayak S, Soumya KV. Abnormal formation and communication of external jugular vein. Int. J. Anat. Var. 2008; 1:15-16. Disponible en: http://www.ijav.org/2008/ ijav 08 015-016.pdf. Acceso en diciembre 05 de 2014.

15. Maskey D, Baral P, Kuwar RB, Rai D, Dhungel S, Jha CB, Bhattacharya S. Unusual venous drainage of face- a case report. Nepal Med Coll J. 2006; 8(4), 286-287. Disponible en: https://www.ncbi.nlm.nih.gov/pubmed/17357652. Acceso en diciembre 05 de 2014

16. Spath P, Barankay AG, Richter JA. Erfahrungen mit der Swan-Ganz-Katheter-Plazierung u"ber die Vena jugularis externa. Anaesthetist. 1985; 34: 367-370.

17. Schwartz AJ, Jobes DR, Levy WJ, Palermo L, Ellison N. Intrathoracic vascular catheterization via the external jugular vein. Anaesthesiology. 1982; 56: 400402. Disponible en: http://anesthesiology.pubs.asahq.org/article.aspx?articleid=1957 023. Acceso en diciembre 06 de 2014.

18. Youngberg JA. Pulmonary artery catheterization via the external jugular vein. South Med J. 1982 Mar;75(3):289-90. https://www.ncbi.nlm.nih.gov/pubmed/7063903

19. Balachandra N, Padmalatha K, Prakash BS, Ramesh BR. Variation of the veins of the head and neck - external jugular vein and facial vein. Int J Anat Var (IJAV).2012;5:99101. Disponible en: http://www.ijav.org/2012/ijav 2012 099-101.pdf. Acceso en diciembre 06 de 2014. 